\title{
Correction: Different impact factors for accurate oscillometric blood pressure measurement between sinus rhythm and atrial fibrillation
}

\author{
Feng Xie $\cdot$ Jinsong $\mathrm{Xu} \cdot$ Huan Liu $\cdot$ Xiaozhong $\mathrm{Li} \cdot$ Yanqing Wu $\cdot$ Hai Su
}

Published online: 30 July 2021

(c) The Author(s), under exclusive licence to Springer Nature Limited 2021

Correction to: Journal of Human Hypertension

https://doi.org/10.1038/s41371-021-00512-9

Unfortunately, an error occurred in the author affiliations. The corrected affiliations are:

Feng Xie \# 1,2, Jinsong Xu \#1, Huan Liu 1, Xiaozhong Li 1, Yanqing Wu 1, Hai Su 3

1 Department of Cardiovascular Medicine, The Second Affiliated Hospital of Nanchang University, Nanchang, China.
2 Working at present in Department of Cardiovascular Medicine, JiangXi Pingxiang People's Hospital, Pingxiang, China.

3 Department of Cardiovascular Medicine, The Second Affiliated Hospital of Nanchang University, Nanchang, China.

The original article has been corrected. 